\title{
Pulse-mode quantum projection synthesis: Effects of mode mismatch on optical state truncation and preparation
}

\author{
Şahin Kaya Özdemir, ${ }^{(a)}$ Adam Miranowicz, ${ }^{(a, b)}$ Masato Koashi, ${ }^{(a)}$ and Nobuyuki Imoto ${ }^{(a, c, d)}$ \\ (a) CREST Research Team for Interacting Carrier Electronics, \\ The Graduate University for Advanced Studies (SOKEN-DAI), Hayama, Kanagawa 240-0193, Japan \\ (b) Nonlinear Optics Division, Institute of Physics, Adam Mickiewicz University, 61-614 Poznań, Poland \\ (c) NTT Basic Research Laboratories, 3-1 Morinosato Wakamiya, Atsugi, Kanagawa 243-0198, Japan \\ (d) Department of Applied Physics, University of Tokyo, 7-3-1 Hongo, Bunkyo-ku, Tokyo 113-8654, Japan
}

(October 25, 2018)

\begin{abstract}
Quantum projection synthesis can be used for phase-probability-distribution measurement, optical-state truncation and preparation. The method relies on interfering optical lights, which is a major challenge in experiments performed by pulsed light sources. In the pulsed regime, the time frequency overlap of the interfering lights plays a crucial role on the efficiency of the method when they have different mode structures. In this paper, the pulsed mode projection synthesis is developed, the mode structure of interfering lights are characterized and the effect of this overlap (or mode match) on the fidelity of optical-state truncation and preparation is investigated. By introducing the positive-operator-valued measure (POVM) for the detection events in the scheme, the effect of mode mismatch between the photon-counting detectors and the incident lights are also presented.
\end{abstract}

\section{INTRODUCTION}

The accurate preparation of quantum states is a crucial task for reliable quantum computation and quantuminformation processing. Several schemes have been proposed for the generation of arbitrary states and their superpositions. One of the most developed systems of state preparation relies on conditional measurement, which brings one of the subsytems of an entangled system to a predetermined state by a measurement on the other subsystem. In these systems, entanglement of the two subsystems is achieved through linear or nonlinear interactions 证田.

The projection-synthesis approach, which has been originally proposed to measure the optical phase probability distribution by Barnett et al. [5], exploits the mixing of two states (one to be measured and the other as reference state) at a beam splitter and a measurement at the output states of the beam splitter [6,7]. This approach, despite of its simplicity, is very flexible to be used for different applications among which we can count the optical state truncation 2,9, 11, preparation of superposition and phase states $[12$ 14 and the teleportation of superposition states [15]. The scheme, which is shown in Fig.1, exploits projection synthesis and is often referred to as Quantum-Scissors Device (QSD) in the applications of optical-state truncation and preparation. It relies on linear optical elements (two beam splitters, BS), a single-photon state, a coherent state and two photon-counting detectors. In the first beam splitter (BS1), single-photon state is mixed with vacuum and an entangled state of one-photon state and vacuum is formed at the output ports of BS1. The state at one of the output ports of BS1 is sent to the second beam splitter (BS2), where it is mixed with the input coherent light to be truncated. The photon-counting detectors placed at the output ports of BS2 count the number of photons incident on them after the action of BS2. The state at the other output port of BS1 is projected on a specific state among many others according to the number of photons counted at the detectors. In the special case of one-photon detection by one of the detectors and none by the other, the output is projected onto a superposition of vacuum and one-photon state which carries the relative phase and amplitude information of the vacuum and one-photon components of the input coherent state.

As is the case for any scheme, where the interference of differently processed light pulses takes place, the characterization of the optical modes of these lights and their effects on the outcome of the experiments is a major challenge for the quantum-scissors device, too. Although it has been shown that the scheme is realizable with the current level of quantum optics technology [9], the studies so far have not considered the problem of mode matching. In this paper, we investigate projection synthesis for quantum-scissors device using the pulse mode formalism and study the effect of mode-mismatch problem on state truncation and preparation.

For the evaluation of the quality of the process, fidelity of the generated state to the desired one is used. Fidelity is a commonly used measure of how close the two states are and is given by

$$
F=\operatorname{Tr}\left[\hat{\rho}_{\text {out }}\left|\phi_{\text {desired }}\right\rangle\left\langle\phi_{\text {desired }}\right|\right],
$$

where $\hat{\rho}_{\text {out }}$ and $\left|\phi_{\text {desired }}\right\rangle$ are the prepared and desired states, respectively. When the prepared state is exactly the desired state then $F=1$, when these two states are orthogonal $F=0$. In practice, the value of fidelity will lie between 0 and 1 , and its value will be a sign of the quality of the process. In general, the quality of the pre- 
pared state strongly depends on the details of the mixing (interference) process and the conditional measurement When these two main phenomena are prone to errors, th generated state may considerably differ from the desirec one.

The paper is organized as follows: In Sec. II, pulsemode formalism is introduced and the calculation of mode mismatch is explicitly shown. The effects of modt mismatch between the interfering lights and the photodetectors are studied in detail in Sec. III, and analytical expressions, which show the mismatch dependence o: fidelity of state truncation by projection synthesis, are given. Then in Sec. IV, the results of the findings are discussed for preparation of arbitrary superposition ot vacuum and one-photon states. A discussion of some practical issues and the characterization of mode structures of fields in a practical scheme are addressed in Sec. V. And finally, Sec. VI includes a brief summary and conclusion of this study.

\section{THEORY OF MODE MISMATCH USING PULSE MODE FORMALISM}

In the QSD scheme shown in Fig.1, interference of vacuum and single-photon states at BS1 (50:50) and that of the entangled state of mode $\hat{b}_{2}$ mode and the coherent state at BS2 (50:50) are the fundamental optical processes. The scheme is usually analyzed in the single mode description in which a pair of annihilation and creation operators for each beam splitter is used. In that picture, the spatio-temporal characteristics of the states input to the beam splitters are assumed to be matched perfectly at the beam splitters and detectors. However, in practice, these interfering lights are prepared independently and thus may have different modes. Moreover, mode definitions of the states at the output of BS2 and that of the measuring apparatus (photon counting detectors) may be different. In these cases, the detection of the correct photon numbers does not mean the correct conditioning (projection) of the desired output state. In practical experiments, high level of attention must be given to match the modes of the input states and the detectors as much as possible for a successful state preparation. A good mode matching shows itself as high visibility and can be a major challenge in experiments.

In this section, we will introduce the pulse-mode formalism and present general expressions to calculate the overlap of two number states with different modes. It is assumed that the bandwidth of the light pulses are sufficiently small and the variation of the beam-splitter transmission and reflection parameters within the pulse bandwidths can be neglected. In this case, these parameters become independent of pulse shape and solely reflect

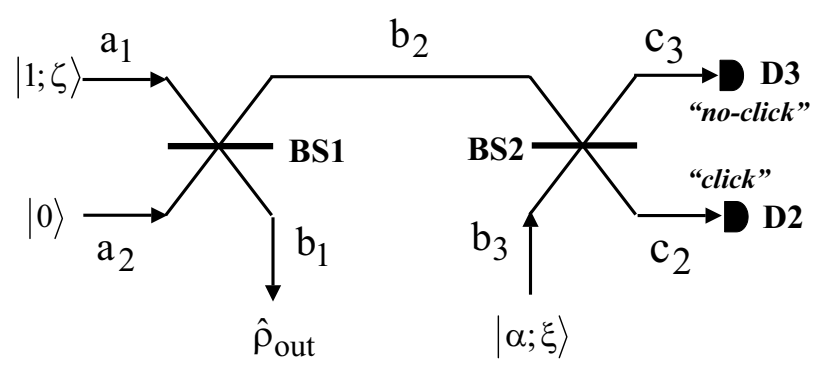

FIG. 1. Schematic configuration of the quantum-scissors device (QSD). BS1, BS2: beam splitters; D2, D3: photon-counting detectors; $|\alpha\rangle,|0\rangle,|1\rangle$ : coherent, vacuum and single-photon states, respectively, $\zeta$ and $\xi$ denote the mode functions of the corresponding input fields and $\rho_{\text {out }}$ is the truncated output state. Desired output state is obtained by one-photon detection at D2 which is denoted as "click" and no photon detection ("no-click") at D3.

the effect of beam splitters; beam splitting will not affect the mode structure of the input light pulses.

Following Refs. [16 19], we define creation and annihilation operators of light pulses in terms of the operators of the monochromatic modes that form them. Then the creation operator for a pulse whose mode profile is described by $\xi$ can be written as

$$
\hat{a}^{\dagger}(\xi)=\int d \omega \xi(\omega) \hat{a}^{\dagger}(\omega)
$$

where $\xi(\omega)$ is normalized as

$$
\|\xi\|^{2} \equiv \int d \omega|\xi(\omega)|^{2}=1 .
$$

Using the continuous-mode bosonic commutators

$$
\left[\hat{a}(\omega), \hat{a}^{\dagger}\left(\omega^{\prime}\right)\right]=\delta\left(\omega-\omega^{\prime}\right)
$$

we can obtain the following commutator

$$
\left[\hat{a}(\xi), \hat{a}^{\dagger}(\xi)\right]=1
$$

In order to analyze the effects of mode mismatch, we have to look at the relation between the mode descriptions of creation operators. The commutator between two operators with different mode descriptions of $\xi(\omega)$ and $\zeta(\omega)$ can be calculated as follows [18]

$$
\begin{aligned}
{\left[\hat{a}(\xi), \hat{a}^{\dagger}(\zeta)\right] } & =\left[\int d \omega \xi^{*}(\omega) \hat{a}(\omega), \int d \omega^{\prime} \zeta\left(\omega^{\prime}\right) \hat{a}^{\dagger}\left(\omega^{\prime}\right)\right] \\
& =\int d \omega \int d \omega^{\prime} \xi^{*}(\omega) \zeta\left(\omega^{\prime}\right)\left[\hat{a}(\omega), \hat{a}^{\dagger}\left(\omega^{\prime}\right)\right] \\
& =\int d \omega \xi^{*}(\omega) \zeta(\omega)=(\xi, \zeta)
\end{aligned}
$$

which means that the commutator between operators of any two modes corresponds to the overlap of these two modes. 
The operators defined above can be used to construct number and coherent states with a given mode description simply by replacing the usual discrete bosonic operators with the pulse-mode operators of the given mode description. In the following, $|\sqcup ; \xi\rangle$ represents a Fock state when $\sqcup$ is a number or written in roman, and a coherent state when $\sqcup$ is written in Greek alphabet. $\xi$ denotes the mode-profile of the corresponding state. Then a number state of mode $\xi$ can be written as

$$
|n ; \xi\rangle=\frac{1}{\sqrt{n !}}\left[\hat{a}^{\dagger}(\xi)\right]^{n}|0\rangle,
$$

and a coherent state as

$$
|\alpha ; \xi\rangle=\exp \left[\alpha \hat{a}^{\dagger}(\xi)-\alpha^{*} \hat{a}(\xi)\right]|0\rangle
$$

with $\hat{a}(\omega)|\alpha ; \xi\rangle=\alpha \xi(\omega)|\alpha ; \xi\rangle$. Here, we define the mode profile function as

$$
g\left(\omega, \omega^{\prime}\right)=\operatorname{Tr}\left[\hat{\rho} \hat{a}^{\dagger}(\omega) \hat{a}\left(\omega^{\prime}\right)\right]
$$

which gives $g\left(\omega, \omega^{\prime}\right)=\zeta\left(\omega, \omega^{\prime}\right)=\zeta(\omega) \zeta^{*}\left(\omega^{\prime}\right)$ for a onephoton state $\hat{\rho}=|1 ; \zeta\rangle\langle 1 ; \zeta|$, and $g\left(\omega, \omega^{\prime}\right)=\xi\left(\omega, \omega^{\prime}\right)=$ $|\alpha|^{2} \xi(\omega) \xi^{*}\left(\omega^{\prime}\right)$ for a single mode coherent state $\hat{\rho}=$ $|\alpha ; \xi\rangle\langle\alpha ; \xi|$ with $\int d \omega \zeta(\omega, \omega)=1$ and $\int d \omega \xi(\omega, \omega)=|\alpha|^{2}$.

The overlap $\langle n ; \xi \mid n ; \zeta\rangle$ of two pure number states $|n ; \zeta\rangle$ and $|n ; \xi\rangle$ can be found by successive applications of Eqs. (2) $-(6)$ :

$$
\begin{aligned}
\langle n ; \xi \mid n ; \zeta\rangle & =\prod_{k=1}^{n} \int d \omega_{k} \xi^{*}\left(\omega_{k}\right) \zeta\left(\omega_{k}\right) \\
& =\prod_{k=1}^{n}(\xi, \zeta)=(\xi, \zeta)^{n}
\end{aligned}
$$

from which the overlap of two pure single photon states can be found as $\langle 1 ; \xi \mid 1 ; \zeta\rangle=(\xi, \zeta)$.

The overlap of a pure one-photon state $|1 ; \xi\rangle$ and a mixed one $\hat{\rho}_{1}$, which is defined as

$$
\hat{\rho}_{1}=\sum_{j} p_{j}\left|1 ; \zeta_{j}\right\rangle\left\langle 1 ; \zeta_{j}\right|
$$

with $\sum_{j} p_{j}=1$, is found using $\operatorname{Tr}\left[\hat{\rho}_{1}|1 ; \xi\rangle\langle 1 ; \xi|\right]$ as

$$
\begin{aligned}
\left\langle 1 ; \xi\left|\hat{\rho}_{1}\right| 1 ; \xi\right\rangle=\int & \int d \omega d \omega^{\prime} \sum_{j} p_{j} \zeta_{j}^{*}\left(\omega^{\prime}\right) \xi\left(\omega^{\prime}\right) \zeta_{j}(\omega) \xi^{*}(\omega) \\
& =\iint d \omega d \omega^{\prime} \zeta\left(\omega, \omega^{\prime}\right) \xi\left(\omega, \omega^{\prime}\right) \\
& =\sum_{j} p_{j}\left|\left(\xi, \zeta_{j}\right)\right|^{2}
\end{aligned}
$$

where $\zeta\left(\omega, \omega^{\prime}\right)=\sum_{j} p_{j} \zeta_{j}(\omega) \zeta_{j}^{*}\left(\omega^{\prime}\right)$, and $\xi\left(\omega, \omega^{\prime}\right)=$ $\xi\left(\omega^{\prime}\right) \xi^{*}(\omega)$ are found using Eq. (9).

The overlap between a mixed number state $\hat{\rho}_{1}$ as given in Eq. (11) and a pure coherent state $|\alpha ; \xi\rangle$ can be found first writing the coherent state in the photon number basis and then applying the above procedure. This will result in

$$
\begin{gathered}
\left\langle\alpha ; \xi\left|\hat{\rho}_{1}\right| \alpha ; \xi\right\rangle=\sum_{j} p_{j} \exp \left[-|\alpha|^{2}\right] \times\left|\left(\zeta_{j}, \xi\right) \alpha\right|^{2} \\
=(\Lambda)^{-1} \iint d \omega d \omega^{\prime} \underbrace{|\alpha|^{2} \xi\left(\omega^{\prime}\right) \xi^{*}(\omega)}_{\xi\left(\omega, \omega^{\prime}\right)} \\
\times \underbrace{\sum_{j} p_{j} \zeta_{j}(\omega) \zeta_{j}^{*}\left(\omega^{\prime}\right)}_{\zeta\left(\omega, \omega^{\prime}\right)} \\
=\frac{\iint d \omega d \omega^{\prime} \xi\left(\omega, \omega^{\prime}\right) \zeta\left(\omega, \omega^{\prime}\right)}{\int d \omega \xi(\omega, \omega) \int d \omega \zeta(\omega, \omega)}
\end{gathered}
$$

with $\Lambda=\int d \omega \xi(\omega, \omega) \int d \omega \zeta(\omega, \omega)=|\alpha|^{2}$. Here we define Eq. (13) as the mode-match parameter $\left|\gamma_{0}\right|^{2}$ which satisfies $\left|\gamma_{0}\right|^{2}+\left|\gamma_{1}\right|^{2}=1$ with $\left|\gamma_{1}\right|^{2}$ representing the modemismatch parameter.

In the following sections, we will use the formalism developed in this section to study the QSD scheme where the input fields are a mixed single photon state and a pure coherent state.

\section{ANALYSIS OF MODE MISMATCH IN QSD SCHEME FOR STATE TRUNCATION}

In an optical-state truncation experiment using the QSD scheme, an input coherent light with an unknown quantum state (intensity and phase) is truncated up to its one-photon state generating, at a remote port, a superposition of its vacuum and one-photon state preserving the relative phase and intensity between these components of the input coherent light.

In this section, we study the state truncation in the pulsed regime and investigate the effect of mode mismatch between the interfering lights on the fidelity of the truncation process. We also consider the case where the mode structures of the photon-counting detectors are different from the mode structures of the light pulses incident on them. We assume that the input coherent light, with unknown quantum state, is at $\hat{b}_{3}$ input of BS2 and has a mode profile described by $\xi$. The $\hat{a}_{1}$ input port of BS1 is fed with a single-photon state whose mode profile is given by $\zeta$. First, we consider the case where the one-photon input to the device is prepared in a mixed state and find the general expressions for the output density operator and fidelity of the process. After the presentation of the general formulas, we will analyze the process, in details, for a single photon prepared in pure state and present the results of this study. In the evaluation of the efficiency of the truncation process in this section, we will impose the condition that the output state should be a superposition of vacuum and one- 
photon states $N(|0\rangle+\alpha|1\rangle)$ in the same mode of the input coherent light $|\alpha\rangle$.

With the single-photon prepared in a mixed state

$$
\hat{\rho}_{a_{1}}=\sum_{j} p_{j}\left|1 ; \zeta_{j}\right\rangle_{a_{1} a_{1}}\left\langle 1 ; \zeta_{j}\right|
$$

with $\sum_{j} p_{j}=1$, and the coherent state as $|\alpha ; \xi\rangle_{b_{3}}$, the overall input to the QSD scheme becomes

$\hat{\rho}_{\text {in }}=\sum_{j} p_{j}\left|1 ; \zeta_{j}\right\rangle_{a_{1} a_{1}}\left\langle 1 ; \zeta_{j}|\otimes| 0\right\rangle_{a_{2} a_{2}}\langle 0|\otimes| \alpha ; \xi\rangle_{b_{3} b_{3}}\langle\alpha ; \xi|$.

Then the state state just before the photon counting can be written as $\hat{\rho}_{\left(b_{1}, c_{2}, c_{3}\right)}=\hat{U}_{2}^{\dagger} \hat{U}_{1}^{\dagger} \hat{\rho}_{\text {in }} \hat{U}_{1} \hat{U}_{2}$ where the actions of the beam splitters BS1 and BS2 are represented by unitary operators $\hat{U}_{1}$ and $\hat{U}_{2}$, respectively [9, 13,20 .

The probability of detecting a "click" at D2 and "noclick" at D3 is given by the trace over the three modes

$$
\mathrm{P}_{10}=\operatorname{Tr}_{\left(b_{1}, c_{2}, c_{3}\right)}\left[\hat{\rho}_{\left(b_{1}, c_{2}, c_{3}\right)} \Pi_{1}^{c_{2}} \Pi_{0}^{c_{3}}\right]
$$

with $\Pi_{1}^{c_{2}}$ and $\Pi_{0}^{c_{3}}$ being the elements of positive-operatorvalued measures (POVMs). In general for a detector with a quantum efficiency of $\eta$, the POVM can be written as

$$
\Pi_{n}=\sum_{m=n}^{\infty} \eta^{n}(1-\eta)^{m-n} C_{n}^{m}|m\rangle\langle m|,
$$

where $n$ and $m$ are the number of detected and incident photons, respectively [21. $C_{n}^{m}$ represent the binomial coefficients, and $\sum_{0}^{\infty} \Pi_{n}=1$. For the sake of simplicity, we assume zero mean dark count $(\nu=0)$ in this study.

Then the output state at $\hat{b}_{1}$ which is conditioned on this detection is found by a partial trace

$$
\hat{\rho}_{\text {out }}=\frac{1}{\mathrm{P}_{10}} \operatorname{Tr}_{\left(c_{2}, c_{3}\right)}\left[\hat{\rho}_{\left(b_{1}, c_{2}, c_{3}\right)} \Pi_{1}^{c_{2}} \Pi_{0}^{c_{3}}\right] .
$$

Then the density operator is written as

$$
\begin{aligned}
& \hat{\rho}_{\text {out }}=\frac{1}{\mathrm{P}_{10}} \sum_{j} p_{j}\left[d_{00}^{(j)}|0\rangle_{b_{1} b_{1}}\left\langle 0\left|+d_{01}^{(j)}\right| 0\right\rangle_{b_{1} b_{1}}\left\langle 1 ; \zeta_{j}\right|\right. \\
& \left.+d_{10}^{(j)}\left|1 ; \zeta_{j}\right\rangle_{b_{1} b_{1}}\left\langle 0\left|+d_{11}^{(j)}\right| 1 ; \zeta_{j}\right\rangle_{b_{1} b_{1}}\left\langle 1 ; \zeta_{j}\right|\right]
\end{aligned}
$$

with the following elements

$$
\begin{aligned}
d_{00}^{(j)} & =\frac{1}{4} c_{c_{2}}\left\langle\lambda ; \xi\left|\hat{c}_{2}\left(\zeta_{j}\right) \Pi_{1}^{c_{2}} \hat{c}_{2}^{\dagger}\left(\zeta_{j}\right)\right| \lambda ; \xi\right\rangle_{c_{2} c_{3}}\left\langle\delta ; \xi\left|\Pi_{0}^{c_{3}}\right| \delta ; \xi\right\rangle_{c_{3}} \\
& +\frac{1}{4} c_{2}\left\langle\lambda ; \xi\left|\Pi_{1}^{c_{2}}\right| \lambda ; \xi\right\rangle_{c_{2} c_{3}}\left\langle\delta ; \xi\left|\hat{c}_{3}\left(\zeta_{j}\right) \Pi_{0}^{c_{3}} \hat{c}_{3}^{\dagger}\left(\zeta_{j}\right)\right| \delta ; \xi\right\rangle_{c_{3}} \\
& +\frac{i}{4} c_{2}\left\langle\lambda ; \xi\left|\hat{c}_{2}\left(\zeta_{j}\right) \Pi_{1}^{c_{2}}\right| \lambda ; \xi\right\rangle_{c_{2} c_{3}}\left\langle\delta ; \xi\left|\Pi_{0}^{c_{3}} \hat{c}_{3}^{\dagger}\left(\zeta_{j}\right)\right| \delta ; \xi\right\rangle_{c_{3}} \\
& -\frac{i}{4} c_{2}\left\langle\lambda ; \xi\left|\Pi_{1}^{c_{2}} \hat{c}_{2}^{\dagger}\left(\zeta_{j}\right)\right| \lambda ; \xi\right\rangle_{c_{2} c_{3}}\left\langle\delta ; \xi\left|\hat{c}_{3}\left(\zeta_{j}\right) \Pi_{0}^{c_{3}}\right| \delta ; \xi\right\rangle_{c_{3}} \\
d_{01}^{(j)} & =\frac{-1}{2 \sqrt{2}} \hat{c}_{2}\left\langle\lambda ; \xi\left|\Pi_{1}^{c_{2}}\right| \lambda ; \xi\right\rangle_{c_{2} c_{3}}\left\langle\delta ; \xi\left|\Pi_{0}^{c_{3}} \hat{c}_{3}^{\dagger}\left(\zeta_{j}\right)\right| \delta ; \xi\right\rangle_{c_{3}} \\
& +\frac{i}{2 \sqrt{2}} c_{2}\left\langle\lambda ; \xi\left|\Pi_{1}^{c_{2}} \hat{c}_{2}^{\dagger}\left(\zeta_{j}\right)\right| \lambda ; \xi\right\rangle_{c_{2} c_{3}}\left\langle\delta ; \xi\left|\Pi_{0}^{c_{3}}\right| \delta ; \xi\right\rangle_{c_{3}} \\
d_{11}^{(j)} & =\frac{1}{2} c_{c_{2}}\left\langle\lambda ; \xi\left|\Pi_{1}^{c_{2}}\right| \lambda ; \xi\right\rangle_{c_{2} c_{3}}\left\langle\delta ; \xi\left|\Pi_{0}^{c_{3}}\right| \delta ; \xi\right\rangle_{c_{3}}
\end{aligned}
$$

and $d_{10}^{(j)}=d_{01}^{(j) *}$ where $\delta=\alpha / \sqrt{2}$ and $\lambda=i \alpha / \sqrt{2}$ are obtained through the action of the BS2 on $|\alpha ; \xi\rangle_{b_{3}}$. The creation operators associated with the outgoing modes of BS2 are represented by $\hat{c}_{k}^{\dagger}$ where $k=2,3$.

Then the fidelity of this output state $\hat{\rho}_{\text {out }}$ to the desired truncated state

$$
\left|\phi_{\text {desired }}\right\rangle=\frac{|0\rangle_{b_{1}}+\alpha|1 ; \xi\rangle_{b_{1}}}{\sqrt{1+|\alpha|^{2}}},
$$

which has the same mode profile $\xi$ of the input coherent light, can be calculated, using Eq. (11), as

$$
F=\frac{\sum_{j} p_{j}\left[d_{00}^{(j)}+2 \operatorname{Re}\left[\alpha \Upsilon_{j} d_{01}^{(j)}\right]+d_{11}^{(j)}|\alpha|^{2}\left|\Upsilon_{j}\right|^{2}\right]}{\left(1+|\alpha|^{2}\right) \sum_{j} p_{j}\left(d_{00}^{(j)}+d_{11}^{(j)}\right)}
$$

where $b_{1}\left\langle 1 ; \zeta_{j} \mid 1 ; \xi\right\rangle_{b_{1}}=\left(\zeta_{j}, \xi\right)=\Upsilon_{j}$ represents the overlap of the mode of the output single-photon state and that of the desired output state. The effect of the overlap of the photon-counting detectors and the fields incident on them is contained in the expressions of the elements of the output density matrix which will be clear in the following subsections.

State truncation using the QSD scheme is based on conditional measurement. Therefore, the correct application and interpretation of photodetection process is essential to evaluate this scheme. In the following subsections, we will present a comparative study of different photon-counting detectors. First, we will use ideal counters which can resolve the photon number incident on them and then proceed with a realistic description of photodetection with conventional photon counters.

\section{A. Photon-number-resolving detectors}

This type of detectors can resolve the number of incident photons. In the following, we will first analyze the scheme for detectors that are matched only to a specific mode and then present the elements of POVM for a more realistic case where the mode of the incident light cannot be resolved.

\section{Mode-resolving detectors}

For mode-resolving detectors, the elements of the POVMs can be written as

$$
\begin{aligned}
& \Pi_{0}^{c_{3}}=\sum_{m=0}^{\infty}(1-\eta)^{m} \hat{P}_{m}^{c_{3}}(\varrho) \\
& \Pi_{1}^{c_{2}}=\sum_{m=0}^{\infty} m \eta(1-\eta)^{m-1} \hat{P}_{m}^{c_{2}}(\varrho)
\end{aligned}
$$


where $\hat{P}_{m}^{c_{k}}(\varrho)$ with $k=2,3$ is the projection onto the eigenspace of $\hat{c}_{k}^{\dagger}(\varrho) \hat{c}_{k}(\varrho)$ with eigenvalue $m$ satisfying the commutators $\left[\hat{P}_{m}^{c_{k}}(\varrho), \hat{c}_{k}^{\dagger}(\xi)\right]=0$ and $\left[\hat{P}_{m}^{c_{k}}(\varrho), \hat{c}_{k}(\xi)\right]=0$ if the overlap $(\xi, \varrho)=0$. Here $\varrho$ represents the light mode that can be resolved by the detectors and $\varrho^{\perp}$ represents the unresolved light mode with $\left(\varrho, \varrho^{\perp}\right)=0$. Then the light modes in Eq. (19) can be decomposed into two orthogonal modes as $\zeta_{j}=\chi_{j} \varrho+\Xi_{j} \varrho_{\zeta_{j}}^{\perp}$ and $\xi=\kappa \varrho+\mu \varrho_{\xi}^{\perp}$ where we define $\chi_{j}=\left(\varrho, \zeta_{j}\right), \Xi_{j}=\left(\varrho_{\zeta_{j}}^{\perp}, \zeta_{j}\right)$ with $\left|\chi_{j}\right|^{2}+\left|\Xi_{j}\right|^{2}=1$ and $\kappa=(\varrho, \xi), \mu=\left(\varrho_{\xi}^{\perp}, \xi\right)$ with $|\kappa|^{2}+|\mu|^{2}=1$ to represent the overlap (mode match) of two modes characterized by $\zeta_{j}$ and $\xi$, with the mode $\varrho$ that can be resolved by the detectors. Consequently, the annihilation and creation operators for a given mode can be decomposed in the same way resulting in $\hat{c_{3}}(\xi)=\kappa^{*} \hat{c_{3}}(\varrho)+\mu^{*} \hat{c_{3}}\left(\varrho_{\xi}^{\perp}\right)$. A similar expression can be obtained for $\hat{c_{2}}\left(\zeta_{j}\right)$ by using the given relations above. The overlap of the modes $\varrho_{\zeta_{j}}^{\perp}$ and $\varrho_{\xi}^{\perp}$ can be found by using the commutators given in Eqs. (5) and (6) as

$$
\left[\hat{c}_{2}\left(\varrho_{\zeta_{j}}^{\perp}\right), \hat{c}_{2}^{\dagger}\left(\varrho_{\xi}^{\perp}\right)\right]=\left(\varrho_{\zeta_{j}}^{\perp}, \varrho_{\xi}^{\perp}\right)=\left(\Upsilon_{j}-\kappa \chi_{j}^{*}\right) /\left(\mu \Xi_{j}^{*}\right) .
$$

Glauber's displacement operator of the form $\hat{D}(\delta ; \xi)$ can be decomposed as $\hat{D}(\delta ; \xi)=\hat{D}(\delta \kappa ; \varrho) \hat{D}\left(\delta \mu ; \varrho_{\xi}^{\perp}\right)$ enabling us to write a coherent state of the form $|\delta ; \xi\rangle$ as $|\delta ; \xi\rangle=\hat{D}(\delta \kappa ; \varrho) \hat{D}\left(\delta \mu ; \varrho_{\xi}^{\perp}\right)|v a c\rangle$. Moreover, from the definition of the $\hat{P}_{m}^{c_{k}}(\varrho)$ operator, we can easily show that $\left[\hat{P}_{m}^{c_{3}}(\varrho), \hat{D}\left(\delta \mu ; \varrho_{\xi}^{\perp}\right)\right]=0$ and $\left[\hat{P}_{m}^{c_{3}}(\varrho), \hat{D}^{\dagger}\left(\delta \mu ; \varrho_{\xi}^{\perp}\right)\right]=0$. The same commutation relation is valid for the displacement operator of mode $\varrho_{\zeta_{j}}^{\perp}$.

Using the elements of POVMs given in Eq. (22) and the transformations

$$
\begin{aligned}
\hat{D}^{\dagger}\left(\delta \mu ; \varrho_{\xi}^{\perp}\right) \hat{c_{3}}\left(\varrho_{\xi}^{\perp}\right) \hat{D}\left(\delta \mu ; \varrho_{\xi}^{\perp}\right) & =\hat{c_{3}}\left(\varrho_{\xi}^{\perp}\right)+\delta \mu \\
\hat{D}^{\dagger}\left(\delta \mu ; \varrho_{\xi}^{\perp}\right) \hat{c_{3}}{ }^{\dagger}\left(\varrho_{\xi}^{\perp}\right) \hat{D}\left(\delta \mu ; \varrho_{\xi}^{\perp}\right) & ={\hat{c_{3}}}^{\dagger}\left(\varrho_{\xi}^{\perp}\right)+\mu^{*} \delta^{*} \\
\hat{D}^{\dagger}\left(\delta \mu ; \varrho_{\xi}^{\perp}\right) \hat{D}\left(\delta \mu ; \varrho_{\xi}^{\perp}\right) & =\hat{D}^{\dagger}(\delta \kappa ; \varrho) \hat{D}(\lambda \kappa ; \varrho)=\hat{\mathcal{I}}
\end{aligned}
$$

where $\hat{\mathcal{I}}$ is the identity operator, together with similar expressions for $\hat{c_{2}}, \lambda$, and $\varrho_{\zeta_{j}}^{\perp}$, we can obtain the following expression

$$
\begin{aligned}
\left\langle\delta ; \xi\left|\Pi_{0}^{c_{3}}\right| \delta ; \xi\right\rangle & =\exp \left[-\eta|\alpha \kappa|^{2} / 2\right] \\
\left\langle\lambda ; \xi\left|\Pi_{1}^{c_{2}}\right| \lambda ; \xi\right\rangle & =\frac{1}{2} \eta|\alpha \kappa|^{2} \exp \left[-\eta|\alpha \kappa|^{2} / 2\right] \\
\left\langle\delta ; \xi\left|\hat{c_{3}}\left(\zeta_{j}\right) \Pi_{0}^{c_{3}}\right| \delta ; \xi\right\rangle & =\frac{1}{\sqrt{2}} \alpha\left(\Upsilon_{j}-\eta \kappa \chi_{j}^{*}\right) \exp \left[-\eta|\alpha \kappa|^{2} / 2\right] \\
\left\langle\lambda ; \xi\left|\hat{c_{2}}\left(\zeta_{j}\right) \Pi_{1}^{c_{2}}\right| \lambda ; \xi\right\rangle & =\frac{i}{2 \sqrt{2}} \eta \alpha\left[2 \kappa \chi_{j}^{*}+|\alpha \kappa|^{2}\left(\Upsilon_{j}-\eta \kappa \chi_{j}^{*}\right)\right] \exp \left[-\eta|\alpha \kappa|^{2} / 2\right] \\
\left\langle\delta ; \xi\left|\hat{c_{3}}\left(\zeta_{j}\right) \Pi_{0}^{c_{3}}{\hat{c_{3}}}^{\dagger}\left(\zeta_{j}\right)\right| \delta ; \xi\right\rangle & =\frac{1}{2}\left[2\left(1-\eta\left|\chi_{j}\right|^{2}\right)+\left|\alpha\left(\Upsilon_{j}-\eta \kappa \chi_{j}^{*}\right)\right|^{2}\right] \exp \left[-\eta|\alpha \kappa|^{2} / 2\right] \\
\left\langle\lambda ; \xi\left|\hat{c_{2}}\left(\zeta_{j}\right) \Pi_{1}^{c_{2}}{\hat{c_{2}}}^{\dagger}\left(\zeta_{j}\right)\right| \lambda ; \xi\right\rangle & =\frac{1}{4} \eta\left[2\left|\chi_{j}\right|^{2}\left(2-\left|\alpha \Upsilon_{j}\right|^{2}-3 \eta|\alpha \kappa|^{2}\right)+|\alpha|^{2}\left(2\left|\kappa+\chi_{j} \Upsilon_{j}\right|^{2}+\left|\alpha \kappa\left(\Upsilon_{j}-\eta \kappa \chi_{j}^{*}\right)\right|^{2}\right)\right] \\
& \times \exp \left[-\eta|\alpha \kappa|^{2} / 2\right] .
\end{aligned}
$$

Equation (25) together with Eq. (19) clearly shows that the output state is dependent on how well the modes of the input lights (single photon and coherent states) are matched to the modes of each other and to the modes of the photon counting detectors.

Using Eq. (25) in Eqs. (17)-(19), and defining the normalization parameter as

$$
\mathcal{N}_{0}=\sum_{j} p_{j}\left[\left|\chi_{j}\right|^{2}+|\alpha \kappa|^{2}\left(2-\eta\left|\chi_{j}\right|^{2}\right)\right]
$$

the output density operator $\hat{\rho}_{\text {out }}$ and the probability of correct detection event $\mathrm{P}_{10}$ can be, respectively, written as

$$
\begin{aligned}
\hat{\rho}_{\text {out }}=\mathcal{N}_{0}^{-1} & \sum_{j} p_{j}\left[\left(|\alpha \kappa|^{2}\left(1-\eta\left|\chi_{j}\right|^{2}\right)+\left|\chi_{j}\right|^{2}\right)|0\rangle_{b 1 b 1}\langle 0|\right. \\
& +\kappa^{*} \alpha^{*} \chi_{j}|0\rangle_{b 1 b 1}\left\langle 1 ; \zeta_{j}\left|+\alpha \kappa \chi_{j}^{*}\right| 1 ; \zeta_{j}\right\rangle_{b 1 b 1}\langle 0| \\
& \left.+|\alpha \kappa|^{2}\left|1 ; \zeta_{j}\right\rangle_{b 1 b 1}\left\langle 1 ; \zeta_{j}\right|\right]
\end{aligned}
$$

and

$$
\mathrm{P}_{10}=\frac{1}{4} \eta \mathcal{N}_{0} \exp \left[-\eta|\alpha \kappa|^{2}\right] .
$$

Then the fidelity of the truncation process can be found using Eqs. (21)-(27) as

$$
F=\frac{\left(1-\eta|\alpha \kappa|^{2}\right) \Gamma_{\chi}+|\alpha|^{2}|\alpha \kappa|^{2} \Gamma_{\Upsilon}+2|\alpha|^{2} \Gamma_{m}+|\alpha \kappa|^{2}}{\left(1+|\alpha|^{2}\right)\left[\left(1-\eta|\alpha \kappa|^{2}\right) \Gamma_{\chi}+2|\alpha \kappa|^{2}\right]}
$$

where we have used

$$
\begin{aligned}
\Gamma_{\chi} & =\sum_{j} p_{j}\left|\chi_{j}\right|^{2}, \quad \Gamma_{\Upsilon}=\sum_{j} p_{j}\left|\Upsilon_{j}\right|^{2} \\
\Gamma_{m} & =\sum_{j} p_{j} \operatorname{Re}\left[\kappa^{*} \Upsilon_{j} \chi_{j}\right] .
\end{aligned}
$$

For the photon-counting detectors that are matched to $\xi$-mode, that is $\varrho=\xi$ implying $\kappa=1$ and $\chi_{j}=\Upsilon_{j}^{*}$, fidelity of the truncation process is found as 


$$
F=1-\frac{|\alpha|^{2}}{1+|\alpha|^{2}} \frac{\left(1+2|\alpha|^{2}\right)-\left|\gamma_{0}\right|^{2}\left(1+|\alpha|^{2}(1+\eta)\right)}{2|\alpha|^{2}+\left|\gamma_{0}\right|^{2}\left[1-\eta|\alpha|^{2}\right]}
$$

where we have defined the mode-match parameter, with the help of Eqs. (12)-(13), as

$$
\left|\gamma_{0}\right|^{2}=\sum_{j} p_{j}\left|\left\langle 1 ; \xi \mid 1 ; \zeta_{j}\right\rangle\right|^{2}=\sum_{j} p_{j}\left|\Upsilon_{j}\right|^{2} .
$$

The density operator can be calculated by substituting Eqs. (32) in Eqs. (27)-(26). For a pure one-photon state input at the $\hat{a}_{1}$ port of BS1, the density matrix simplifies into

$$
\hat{\rho}_{\text {out }}=\mathcal{N}_{1}^{-1}\left(\begin{array}{cc}
|\alpha|^{2}+\left(1-\eta|\alpha|^{2}\right)\left|\gamma_{0}\right|^{2} & \alpha^{*} \gamma_{0}^{*} \\
\alpha \gamma_{0} & |\alpha|^{2}
\end{array}\right)
$$

where $\mathcal{N}_{1}=2|\alpha|^{2}+\left(1-\eta|\alpha|^{2}\right)\left|\gamma_{0}\right|^{2}$ and $\left|\gamma_{0}\right|^{2}=|(\zeta, \xi)|^{2}$.

If the detectors can resolve only the mode of the onephoton state, $\left(\varrho=\zeta_{j}\right)$, the expression for fidelity can be obtained from Eq. (29) by substituting $\chi_{j}=1$ and $\kappa=\Upsilon_{j}$. When the mode-mismatch parameter $\left(\left|\gamma_{1}\right|^{2}=1-\left|\gamma_{0}\right|^{2}\right)$, equals to one, the fidelity of truncation becomes $1 /\left(1+|\alpha|^{2}\right)$ independent of the detection efficiency, solely dependent on the intensity of the input coherent light to be truncated. With increasing $|\alpha|^{2}$, fidelity of truncation decreases. On the other hand, $P_{10}$ will take the value $\eta / 4$ independent of $|\alpha|^{2}$. For the special case of $\eta=1$, the expression for fidelity simplifies to

$$
F=1-\frac{\left|\alpha \gamma_{1}\right|^{2}}{1+|\alpha|^{2}}
$$

where the linear dependence of fidelity on modemismatch parameter is clearly seen.

The density matrix of the truncated output state, when the input one-photon state is a pure one, becomes

$$
\hat{\rho}_{\text {out }}=\mathcal{N}_{2}^{-1}\left(\begin{array}{cc}
1+(1-\eta)\left|\alpha \gamma_{0}\right|^{2} & \alpha^{*} \gamma_{0}^{*} \\
\alpha \gamma_{0} & \left|\alpha \gamma_{0}\right|^{2}
\end{array}\right)
$$

with $\mathcal{N}_{2}=1+(2-\eta)\left|\alpha \gamma_{0}\right|^{2}$. The density matrix given in Eq. (35) shows that mode-match parameter affects both the diagonal and off-diagonal terms of the density matrix as well as the probability of proper detection $P_{10}$. From Eq. (27), it is clearly seen that when $\varrho$ is set to $\zeta$, the amplitude of the input coherent state $|\alpha ; \xi\rangle$ is re-scaled with the amount of overlap between $\xi$ and $\zeta$ modes. This corresponds to the case where an input of the form $\left|\alpha \gamma_{0} ; \zeta\right\rangle$ is used as the input coherent state.

If the detectors cannot resolve the mode $\zeta_{j}$ of the onephoton state, that is $\zeta_{j}=\varrho_{\zeta_{j}}^{\perp}$ hence $\chi_{j}=0$, the output state becomes independent of the photon counting process which results in a classical mixture of vacuum and one-photon states. On the other hand, when $\xi=\varrho_{\xi}^{\perp}$ with $\kappa=0$, the quantum state at the output will be vacuum because the detected photon will always originate from the input single photon state.

\section{Mode-unresolving detectors}

Since the detectors cannot resolve the mode, photons of any mode are registered by the detectors. In a onephoton detection event, the registered photon could have been in either of the modes. A no-photon detection event would imply that detector has not registered any photon of neither of the modes. Then the elements of the POVM can be written as in Eq. (22) with $\hat{P}_{m}^{c_{k}}(\varrho)$ replaced by $\hat{P}_{m}^{c_{k}}$ resulting in

$$
\begin{aligned}
& \Pi_{0}^{c_{3}}=\sum_{m=0}^{\infty}(1-\eta)^{m} \hat{P}_{m}^{c_{3}} \\
& \Pi_{1}^{c_{2}}=\sum_{m=0}^{\infty} m \eta(1-\eta)^{m-1} \hat{P}_{m}^{c_{2}}
\end{aligned}
$$

where $\hat{P}_{m}^{c_{k}}$ is the projection operator to the subspace with $m$ photons in total. Consequently, the output density operator and the probability of correct detection event $P_{01}$ become

$$
\begin{aligned}
\hat{\rho}_{\text {out }}= & \mathcal{N}_{3}^{-1} \sum_{j} p_{j}\left[\left[1+|\alpha|^{2}(1-\eta)\right]|0\rangle_{b 1 b 1}\langle 0|\right. \\
& +\alpha^{*} \Upsilon_{j}^{*}|0\rangle_{b 1 b 1}\left\langle 1 ; \zeta_{j}\left|+\alpha \Upsilon_{j}\right| 1 ; \zeta_{j}\right\rangle_{b 1 b 1}\langle 0| \\
& \left.+|\alpha|^{2}\left|1 ; \zeta_{j}\right\rangle_{b 1 b 1}\left\langle 1 ; \zeta_{j}\right|\right]
\end{aligned}
$$

and

$$
P_{10}=\frac{1}{4} \eta \mathcal{N}_{3} \exp \left(-\eta|\alpha|^{2}\right)
$$

with $\mathcal{N}_{3}=1+|\alpha|^{2}(2-\eta)$. Then the fidelity of truncation can be calculated from Eqs. (21) and (37) as

$$
F=1-\frac{|\alpha|^{2}\left[2+|\alpha|^{2}(2-\eta)-\left|\gamma_{0}\right|^{2}\left(2+|\alpha|^{2}\right)\right]}{\left(1+|\alpha|^{2}\right)\left[1+|\alpha|^{2}(2-\eta)\right]}
$$

where we have used $\left|\gamma_{0}\right|^{2}=\sum_{j} p_{j}\left|\Upsilon_{j}\right|^{2}$ from which the amount of mode-mismatch is calculated as $\left|\gamma_{1}\right|^{2}=$ $1-\left|\gamma_{0}\right|^{2}$. For this type of detectors, it is observed that (i) mode mismatch between the single photon input and the coherent input affects only the off-diagonal elements of the output density matrix, (ii) fidelity of the truncation process decreases linearly with increasing $\left|\gamma_{1}\right|^{2}$, (iii) the rate of decrease in the fidelity with respect to $\left|\gamma_{1}\right|^{2}$ is higher for higher values of $\eta$ at a constant $|\alpha|^{2}$, and (iv) for $\left|\gamma_{1}\right|^{2} \leq\left(1+|\alpha|^{2}\right) /\left(2+|\alpha|^{2}\right)$, increasing $\eta$ can partially compensate the mode-mismatch effect on the fidelity and increase the value of fidelity, however for higher $\left|\gamma_{1}\right|^{2}$ values, increasing $\eta$ causes slight decrease in fidelity. 


\section{B. Conventional photodetectors}

Conventional photodetectors (CPs) that are available in the market cannot perform the ideal measurement of photon number counting. The avalanche process taking place in the photodetectors makes it difficult to discriminate between the presence of single photon and more photons. The outcomes of such a detector can be either "YES", when any number of photons are incident on the photodetector and cause a "click" or "NO" when no photons are detected. Moreover, CPs cannot resolve the mode of the incoming photon and thus show a "click" for photons belonging to any mode. Then for the QSD scheme, where there are lights with different mode profiles incident on the detectors, (a)

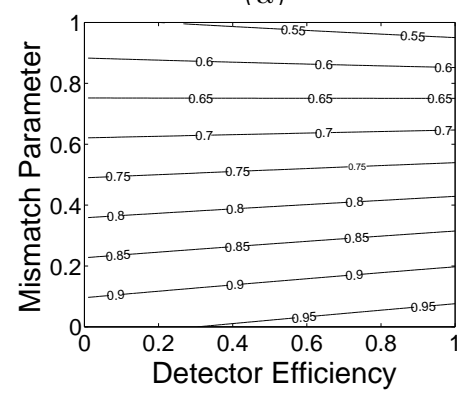

(b)

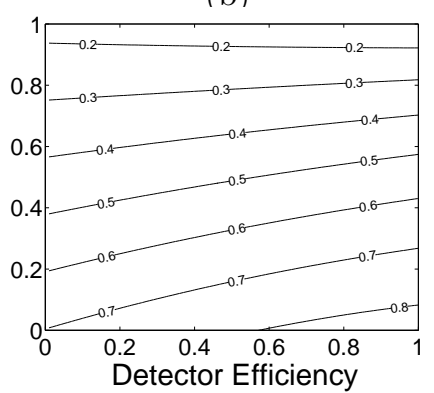

FIG. 2. Effect of mode-mismatch parameter $\left|\gamma_{1}\right|^{2}$ and the detector efficiency $\eta$ on the fidelity of state truncation using conventional photon detectors. The intensity of input coherent light to be truncated are (a) $|\alpha|^{2}=0.4$, and (b) $|\alpha|^{2}=2.5$.

the measurement can be described by $\Pi_{0}^{c 3}$ which is the same as given in (36), and by $\Pi_{1}^{c 2}$ that can be written as

$$
\begin{aligned}
\Pi_{1}^{c 2} & =1-\Pi_{0}^{c 2} \\
& =1-\sum_{m=0}^{\infty}(1-\eta)^{m} \hat{P}_{m}^{c_{2}} .
\end{aligned}
$$

Consequently, the elements of the density operator for the generated output state, and $P_{10}$ are found as

$$
\begin{aligned}
\hat{\rho}_{\text {out }}= & \sum_{j} p_{j}\left[\left(2(2-\eta)+\left|\eta \alpha \Upsilon_{j}\right|^{2}-4 x(1-\eta)\right)|0\rangle_{b 1 b 1}\langle 0|\right. \\
& +2 \eta \alpha^{*} \Upsilon_{j}^{*}|0\rangle_{b 1 b 1}\left\langle 1 ; \zeta_{j}\left|+2 \eta \alpha \Upsilon_{j}\right| 1 ; \zeta_{j}\right\rangle_{b 1 b 1}\langle 0| \\
& \left.+4(1-x)\left|1 ; \zeta_{j}\right\rangle_{b 1 b 1}\left\langle 1 ; \zeta_{j}\right|\right] \times \mathcal{N}_{4}^{-1},
\end{aligned}
$$

and

$$
P_{10}=\frac{x}{8} \mathcal{N}_{4}
$$

where $\mathcal{N}_{4}=\sum_{j} p_{j}\left[2[4(1-x)+\eta(2 x-1)]+\left|\eta \alpha \Upsilon_{j}\right|^{2}\right]$ and $x=\exp \left(-\eta|\alpha|^{2} / 2\right)$. Then the fidelity of the truncation process can be calculated using (21) as

$$
\begin{aligned}
F=1-\frac{1}{\left(1+|\alpha|^{2}\right) \mathcal{N}_{4}}\{4(1-x) \\
+|\alpha|^{2}[2(4-\eta)-4 x(2-\eta) \\
\left.\left.\quad+\left|\gamma_{0}\right|^{2}\left(|\alpha|^{2} \eta^{2}-4(1-x+\eta)\right)\right]\right\} .
\end{aligned}
$$

If the intensity of the coherent light to be truncated is $|\alpha|^{2}=1$, fidelity of the process for $\eta=1$ drops from 0.94 to 0.28 when $\left|\gamma_{1}\right|^{2}$ increases from zero (perfect match between the lights) to one (complete mismatch). In the same way, probability of correct detection events drops from $\sim 0.35$ to $\sim 0.27$. When the density matrix is analyzed for this condition, it is seen that for the complete mismatch case, the off-diagonal elements become zero and the output state is a classical mixture of vacuum and one-photon states, $0.56|0\rangle\langle 0|+0.44| 1\rangle\langle 1|$.

In Fig. 2, we have depicted constant-fidelity contours as a function of $\left|\gamma_{1}\right|^{2}$ and $\eta$ for state truncation using conventional photon counters. It is seen that for low intensity input coherent light, the effect of the mismatch on the fidelity of the truncation process is more profound than that of the detector efficiency $\eta$. Effect of $\eta$ on the value of fidelity and the allowable range of mode mismatch is more significant for higher values of $|\alpha|^{2}$ than the smaller values. The amount of mode-mismatch that can be tolerated to achieve a predetermined constant fidelity $F$, is much higher for low intensity input coherent light than that of the high intensity coherent light.

\section{MODE-MISMATCH EFFECTS ON STATE PREPARATION BY QSD}

Quantum-scissors device which exploits projection synthesis can be used not only for state truncation but preparation of arbitrary superposition of vacuum and one photon states, $N_{\kappa}\left[\kappa_{0}|0\rangle+\kappa_{1}|1\rangle\right]$, as well, where $N_{\kappa}=1 / \sqrt{\left|\kappa_{0}\right|^{2}+\left|\kappa_{1}\right|^{2}}$. This can be achieved with high fidelity and nonzero probability by properly choosing the intensity of the input coherent light. State preparation using QSD scheme differs from the state truncation with the condition that in state truncation quantum state of the input coherent light is not known, however in state preparation the quantum state of the input coherent light is optimized to prepare a known desired state.

For photon number resolving detectors for which the elements of POVM are given in Eq. 36. , the highest fidelity to the desired state may not necessarily be obtained at $\alpha=\kappa_{1} / \kappa_{0}$ due to both the nonunit detector efficiency and the mode mismatch between the input states. So, we do not fix $\alpha$ to be $\kappa_{1} / \kappa_{0}$ but leave it as a parameter to be optimized for the highest fidelity. Then the fidelity of this state preparation is found as

$$
F=\frac{1+2|\alpha \beta|\left|\gamma_{0}\right|^{2}+\left|\alpha \beta \gamma_{0}\right|^{2}+(1-\eta)|\alpha|^{2}}{\left(1+|\beta|^{2}\right)\left[1+(2-\eta)|\alpha|^{2}\right]}
$$


where we have assumed that $\arg (\alpha)=\arg (\beta)$ with $\beta=\kappa_{1} / \kappa_{0}$. Optimum value of $|\alpha|$ which maximizes the fidelity of state preparation for an arbitrary $\beta$ is found as

$|\alpha|=\left[\frac{\left(\left|\beta \gamma_{0}\right|^{2}-1\right)^{2}}{4|\beta|^{2}\left|\gamma_{0}\right|^{4}(2-\eta)^{2}}+\frac{1}{(2-\eta)}\right]^{1 / 2}+\frac{\left|\beta \gamma_{0}\right|^{2}-1}{2|\beta|\left|\gamma_{0}\right|^{2}(2-\eta)}$

It is seen that the increase in $\eta$ shifts the optimized value of $|\alpha|$ to higher values. As the mode mismatch increases, the optimized value of $|\alpha|$ decreases, and in the limiting case $\left|\gamma_{0}\right|^{2}=0\left(\left|\gamma_{1}\right|^{2}=1\right)$, it becomes zero independent of $\eta$ and $|\beta|$.

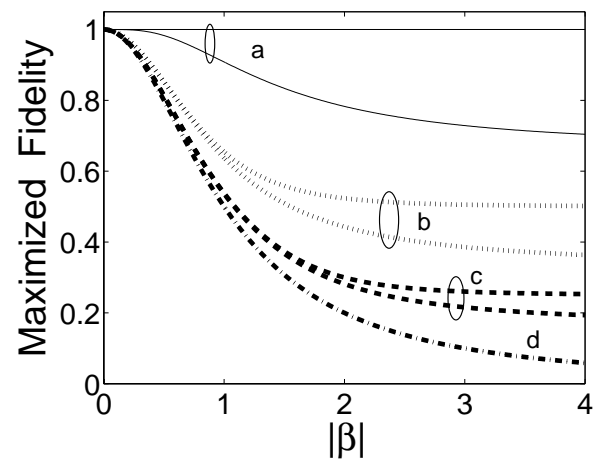

FIG. 3. Effect of mode-mismatch parameter on the maximum fidelity of state preparation with detectors of $\eta=0.5$ and $\eta=1.0$ when $\left|\gamma_{1}\right|^{2}$ equals: a, 0 (perfectly matched modes), b, 0.50, c, 0.75 and d, 1.0 (complete mismatch). Fidelity obtained for $\eta=1.0$ is higher than the fidelity for $\eta=0.5$.

Figures 3 and 4 show the results of this study for which it is understood that the relative weight of vacuum and one-photon state in the superposition is crucial for the amount of mode mismatch that can be tolerated for state preparation with high fidelity. If $|\beta| \leq 0.4$, fidelity values of $F \geq 0.9$ for any amount of mode mismatch with $\eta=0.5$. In this range of $|\beta|$, increasing $\eta$ does not cause a significant improvement on the value of fidelity. The effect of $\eta$ and $\left|\gamma_{1}\right|^{2}$ is more profound when the weight of one-photon component is dominant in the superposition, that is when $|\beta|>1.0$. To illustrate this we consider the preparation of states with $|\beta|$ equals 0.2 and 5 . With $\eta=0.5$ and $\left|\gamma_{1}\right|^{2}=0.5$, the corresponding maximized fidelities are found as 0.97 and 0.35 . When $\eta$ is increased to 1.0 , the fidelities will be 0.97 (no change) and 0.50 , respectively. On the other hand, for $\eta=0.5$, increasing the mismatch parameter $\left|\gamma_{1}\right|^{2}$ from 0.5 to 0.8 will cause a decrease in the fidelities which will become 0.96 and 0.15 , respectively.

For the case of conventional photon counters as the detectors, the expression of the optimized $|\alpha|$ and $F$ are too lengthy and complicated to give here. To have an idea of the state preparation with such detectors, here we give some numerical values: To prepare a state with $|\beta|=0.4$, the optimum value for $|\alpha|$ and the corresponding maximized fidelity are 0.39 and 0.99 when modes are completely matched and $\eta=0.7$. However, with increasing mode mismatch amount to $\left|\gamma_{1}\right|^{2}=0.25$ and 0.5 , the optimized $|\alpha|$ values decrease to 0.3 and 0.21 with the corresponding fidelity values of 0.94 and 0.90 , respectively. Further increase in the amount of mode mismatch forces the optimized $|\alpha|$ to become much closer to zero and the fidelity to the minimum value of 0.862 . For $\eta=0.5$, the desired state can still be prepared with a fidelity $\geq 0.90$ if the mode mismatch is kept below 0.5 for which the optimized $|\alpha|$ will lie in the range $0.21 \leq|\alpha| \leq 0.38$. Then it can be said that superposition states, for which the

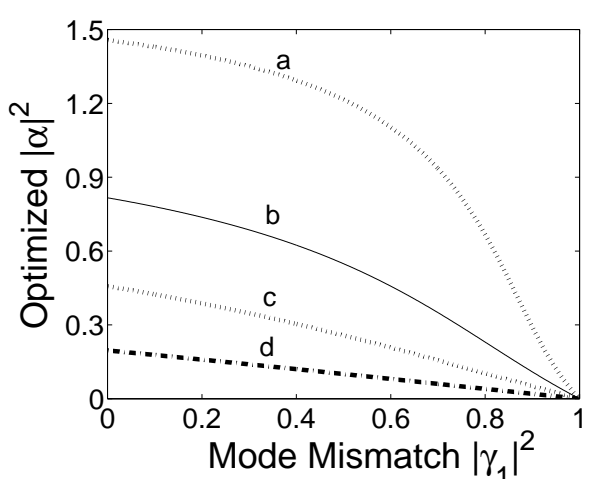

FIG. 4. Effect of mode mismatch parameter on the optimized intensity of the input coherent light $|\alpha|^{2}$ at which fidelity of state preparation is maximized for $|\beta|$ equals to: a, $0.2, \mathrm{~b}, 0.5, \mathrm{c}, 1.0, \mathrm{~d}, 2$. A detection efficiency of $\eta=0.5$ is assumed.

vacuum component of the superposition is dominant, can be prepared with high fidelity even with mode mismatch as much as $50 \%$. On the other hand, when the onephoton component of the state becomes dominant, the mode mismatch show a much higher deteriorating effect on the fidelity of state preparation. When $|\beta|=2.5$ (the weights of vacuum and one-photon states are interchanged), an increase of mode mismatch from zero to 0.25 causes the optimized value of $|\alpha|$ to change from 1.68 to 1.97 . This in turn, causes the maximized fidelity to decrease from 0.71 to 0.58 which is a $18.3 \%$ decrease much higher than the decrease of $4.26 \%$ of the case $|\beta|=0.4$.

\section{MODE STRUCTURES OF FIELDS AND PRACTICAL CONSIDERATIONS}

At the input of the BS2, one of the interfering fields is the input coherent light and the other field is either a single-photon wave packet or vacuum. In case of vacuum, the mode match is not a problem. However, when the field is single-photon then its mode must be matched as much as possible to the mode of the input coherent light. It is seen in Eqs. (41)-(43) that the fidelity 
of truncation process using conventional photodetectors is a function of detector efficiency $\eta$, intensity $|\alpha|^{2}$ of the coherent light to be truncated and the overlap of the modes of one-photon state and the coherent state which is defined as $\left|\gamma_{0}\right|^{2}=\sum_{j} p_{j}\left|\Upsilon_{j}\right|^{2}$ when one-photon is in a mixed state of the form given in Eq. (11). Then in a practical scheme, where $\eta$ is limited with the CPs being used and $|\alpha|^{2}$ can be set freely, an information on the value of $\left|\gamma_{0}\right|^{2}$ will enable the calculation of fidelity to evaluate the efficiency and the quality of the truncation process. Once the mode profiles of the input lights are characterized correctly, their overlap can be found easily using Eq. (13) and consequently, fidelity of the process can be calculated using Eq. (43). Therefore, to have an idea on the bounds of mode matching and the physical phenomena affecting the process, the characterization of the mode structures of the coherent state $|\alpha ; \xi\rangle$ and the single-photon state $|1 ; \zeta\rangle$ is crucial.

In practice, the single-photon state input to the QSD scheme is prepared by a conditional measurement on a biphoton state generated by pulsed spontaneous parametric down conversion (SPDC). In this process, a pump beam converts spontaneously, with a small probability $\mathcal{O}\left(10^{-4}\right)$, into two photons with lower energy due to an interaction in a nonlinear crystal. The two photons, which are created almost simultaneously within a time window that is given by the inverse of the emitted bandwidth, constitute a highly entangled quantum state and are separated into two emission channels which are named as idler and signal. Starting from the interaction Hamiltonian of SPDC, the biphoton state generated by a pulsed light can be written as 22 25]

$$
\begin{aligned}
|\varphi\rangle= & |0\rangle_{i}|0\rangle_{s}-\mathrm{i} \chi \int d^{3} k_{s} d^{3} k_{i} d \omega_{s} d \omega_{i}\left|1 ; k_{i}, \omega_{i}\right\rangle_{i}\left|1 ; k_{s}, \omega_{s}\right\rangle_{s} \\
& \times \int d^{3} k_{p} d \omega_{p} E_{p}^{(+)}\left(\mathbf{k}_{p}, \omega_{p}\right) \int_{t_{0}}^{\infty} d t \mathrm{e}^{\mathrm{i}\left(-\omega_{p}+\omega_{i}+\omega_{s}\right) t} \\
& \times \int d^{3} r \mathbf{K}(\mathbf{r}) \mathrm{e}^{\mathrm{i}\left(\mathbf{k}_{p}-\mathbf{k}_{s}-\mathbf{k}_{i} \mathbf{r}\right)}+h . c .
\end{aligned}
$$

where $E_{p}^{(+)}\left(\mathbf{k}_{p}, \omega_{p}\right)$ is the positive-frequency electric field operator of the pump, $\chi$ is proportional to the second order nonlinear susceptibility and $\mathbf{K}(\mathbf{r})$ describes the volume of the nonlinear crystal with a value of one inside the crystal and zero outside. The last integral corresponds to the Fourier transform of $\mathbf{K}(\mathbf{r})$ which can be written as $\mathbf{K}(\Delta \mathbf{k})$ with $\Delta \mathbf{k}=\mathbf{k}_{p}-\mathbf{k}_{s}-\mathbf{k}_{i}$ and has the form of sinc-function. To simplify the calculations the following assumptions are made: (i) limit of the time integration in Eq. (46) can be taken from $-\infty$ to $+\infty$ because we are interested in the fields far from the crystal, thus integral becomes an impulse function $\delta\left(\omega_{p}-\omega_{s}-\omega_{i}\right)$ expressing the energy conservation in the process, (ii) crystal volume is much larger than the spatial extent of the pump pulse inside the crystal, thus sinc-functions can be approximated by impulse function expressing perfect phase matching, (iii) we confine ourselves to a single spatial mode thus replace k-integrals by frequency integrals, and (iv) the pump is a pure strong coherent light. After straightforward but lengthy calculations, we end up with the following biphoton state

$$
|\varphi\rangle=|0\rangle_{i}|0\rangle_{s}-\mathrm{i} \chi \int E_{p}^{(+)}\left(\omega_{s}+\omega_{i}\right) d \omega_{s} d \omega_{i}\left|1 ; \omega_{i}\right\rangle_{i}\left|1 ; \omega_{s}\right\rangle_{s},
$$

The detection of a photon in the idler channel projects the quantum state in the signal channel into a one-photon state. The photon in the idler channel is selected by spatial and frequency filters which determine the mode structure of detected photon, this, in turn, will affect the mode structure of the photon in the signal channel which is conditioned on the detection in the idler channel. Here, since only a single spatial mode is considered we focus on the effect of the characteristics of temporal filters on the process. Filtering operator $\hat{\mathcal{F}}_{i}$ which selects the photon in the idler channel is written as

$$
\hat{\mathcal{F}}_{i}=\int d \omega_{i} F\left(\omega_{i}\right)\left|1 ; \omega_{i}\right\rangle_{i, i}\left\langle 1 ; \omega_{i}\right| .
$$

where $F\left(\omega_{i}\right)$ denotes the transmission function of the filter. Then the unnormalized state in the signal channel becomes $\operatorname{Tr}_{i}\left(\hat{\mathcal{F}}_{i}|\varphi\rangle\langle\varphi|\right)$ where trace is taken over idler states. Consequently, the field in the signal channel can be found as

$$
\begin{aligned}
& \hat{\rho}_{s}= \operatorname{Tr}_{\mathrm{i}}\left(\hat{\mathcal{F}}_{i}|\varphi\rangle\langle\varphi|\right) \\
&=|\chi|^{2} \int d \omega_{s} d \omega_{s}^{\prime} d \omega_{i} F\left(\omega_{i}\right) \\
& \quad E_{p}^{(-)}\left(\omega_{s}+\omega_{i}\right) E_{p}^{(+)}\left(\omega_{s}^{\prime}+\omega_{i}\right)\left|1 ; \omega_{s}\right\rangle_{s s}\left\langle 1 ; \omega_{s}^{\prime}\right| .
\end{aligned}
$$

In a practical QSD application, this one-photon state is input to BS1 after which it interferes with the coherent state at BS2. We assume that a collimated pump field with a Gaussian spectral distribution

$$
E_{p}^{(+)}(\omega)=E_{0} \exp \left[-\frac{\left(\omega-\omega_{p}^{o}\right)^{2}}{2 \sigma_{p}^{2}}\right],
$$

and a Gaussian spectral filter in the idler channel with an intensity transmission function

$$
F\left(\omega_{i}\right)=F_{0} \exp \left[-\frac{\left(\omega-\omega_{i}^{o}\right)^{2}}{\sigma_{i}^{2}}\right],
$$

where $2 \sqrt{2} \sigma_{p}$ and $2 \sigma_{i}$ are the $1 / e$-widths of the pump field and the intensity transmission function of the interference filter in the idler channel with the central frequencies of $\omega_{p}^{o}$ and $\omega_{i}^{o}=\omega_{p}^{o} / 2$. Then using (49)-(51), the state in the signal channel is found as [25] 


$$
\begin{array}{r}
\hat{\rho}_{s}=\Gamma_{0} \int d \omega_{s} d \omega_{s}^{\prime} \exp \left[-\frac{\Delta X_{1}^{2}+\Delta X_{2}^{2}}{2\left(\sigma_{p}^{2}+\sigma_{i}^{2}\right)}-\frac{\sigma_{i}^{2}\left(\omega_{s}-\omega_{s}^{\prime}\right)^{2}}{4 \sigma_{p}^{2}\left(\sigma_{p}^{2}+\sigma_{i}^{2}\right)}\right] \\
\otimes\left|1 ; \omega_{s}\right\rangle_{s s}\left\langle 1 ; \omega_{s}^{\prime}\right|,
\end{array}
$$

where $\Gamma_{0}$ is a constant factor, $\Delta X_{1}=\omega_{s}-\omega_{p}^{0}+\omega_{i}^{0}$ and $\Delta X_{2}$ is the same as $\Delta X_{1}$ with $\omega_{s}$ replaced by $\omega_{s}^{\prime}$. A comparison of Eq. (52) with Eq. (9) will reveal that the term $\Gamma_{0}$ multiplied by the exponential corresponds the mode profile, $\zeta\left(\omega, \omega^{\prime}\right)$ of the state in the signal channel.

The light to be truncated in the QSD scheme is in a coherent state and it is taken from the same pulsed laser before it is frequency doubled to obtain the pump pulse of $\omega_{p}^{0}$ for SPDC. Then the coherent state to be truncated has a spectrum with a central frequency $\omega_{c}=\omega_{p}^{0} / 2$. Mode profile of the coherent light can be found by taking its correlation function which will yield

$$
\xi\left(\omega, \omega^{\prime}\right)=\Gamma_{1} \exp \left[-\frac{\left(\omega-\omega_{c}\right)^{2}+\left(\omega^{\prime}-\omega_{c}\right)^{2}}{2 \sigma_{c}^{2}}\right] .
$$

Assuming that the beam splitters and the propagation of the fields until they mix at BS2 do not change the mode profile of the fields, the overlap of the one-photon state and the coherent state can be found using

$$
\left|\gamma_{0}\right|^{2}=\frac{\int d \omega d \omega^{\prime} \xi\left(\omega, \omega^{\prime}\right) \zeta\left(\omega, \omega^{\prime}\right)}{\int d \omega \xi(\omega, \omega) \int d \omega \zeta(\omega, \omega)}
$$

as in Eq. (13).

If we assume that for each run of the experiment, the mode-profile of the photon in the signal channel is the same and reproducible, the mode-match parameter can be expressed by the following simple expression

$$
\left|\gamma_{0}\right|^{2}=\frac{2 \sigma_{c} \sigma_{p}}{\left(\sigma_{c}^{2}+\sigma_{p}^{2}\right)} \frac{1}{\sqrt{1+\left(\sigma_{i}^{2}\right) /\left(\sigma_{c}^{2}+\sigma_{p}^{2}\right)}} .
$$

This expression gives the lower theoretical bound for the temporal mode match for a QSD realization.

In practice, the data related with the filters are given as full-width-half-maximum (FWHM) of the intensity transmission function (intensity versus wavelength) and we measure the FWHM of the intensity spectrum of the light field. Therefore, we have to calculate the modematch parameter using these experimentally accessible data. Applying the narrow-bandwidth limit $\sigma_{i, p} \ll \omega_{i, p}^{o}$ and $\sigma_{c} \ll \omega_{c}$, the relation between $\sigma_{\ell}$ of the functions in Eqs. (50)-(53) and the experimentally accessible bandwidths $\left(\lambda_{\ell, F W H M}\right)$ with $\ell=c, i, p$ is given as $\sigma_{\ell}=\sqrt{1 / \ln 2} \pi c\left(\Delta \lambda_{\ell, F W H M} / \lambda_{\ell}^{2}\right)$ with $\lambda_{\ell}$ being the central wavelength of the spectrum. As a preliminary experiment, for example, we have measured full-width at halfmaximum (FWHM) bandwidths as $\Delta \lambda_{c, F W H M}=7 \mathrm{~nm}$ for a pulsed laser with central frequency $\lambda_{c}=790 \mathrm{~nm}$ and $\Delta \lambda_{p, F W H M}=4 \mathrm{~nm}$ after it is frequency doubled. With these values, the lower bound for mode match is found as $\left|\gamma_{0}\right|_{L B}^{2} \simeq 0.64,0.72$ and 0.73 , respectively, for interference filters of $\lambda_{i, F W H M}=10 \mathrm{~nm}, 4 \mathrm{~nm}$, and $1 \mathrm{~nm}$ used in the idler channel. It is clearly seen that narrow-band filtering in the idler channel is crucial for high values of mode overlap. The overlap of the spectrum of the modes can be further increased by using interference filter between BS2 and the photon-counting detectors as in the scheme in [9]. In that case, the problem reduces to first filtering the signal and the coherent light spectrum with the same narrow-band filter and then calculate their overlap. Then for a filter of the form (51) with a $1 / e$-width of $2 \sigma_{f}$, theoretical upper bound for overlap is calculated as

$$
\left|\gamma_{0}\right|^{2}=\frac{2 \sqrt{\mu_{c} \mu_{p}\left(1+2 \mu_{c}\right)} \sqrt{1+2\left(\mu_{p}+\mu_{i}\right)}}{\left(\mu_{c}+\mu_{p}+4 \mu_{c} \mu_{p}\right) \sqrt{1+\frac{2 \mu_{i}\left(1+4 \mu_{c}\right)}{\left(\mu_{c}+\mu_{p}+4 \mu_{c} \mu_{p}\right)}}},
$$

where $\mu_{k}=\left(\sigma_{k} / \sigma_{f}\right)^{2}$ with $k=i, c, p$. In the limiting cases: (a) $\sigma_{f} \rightarrow \infty$, this expression becomes equal to (55), (b) $\sigma_{f} \rightarrow 0$, the $\left|\gamma_{0}\right|^{2}$ approaches one. Using the numerical values given above, if $\sigma_{f}=\sigma_{i}=10 \mathrm{~nm}$ is chosen, $\left|\gamma_{0}\right|^{2} \simeq 0.83$ is obtained. For $\sigma_{f}=\sigma_{i}=4 \mathrm{~nm},\left|\gamma_{0}\right|^{2}$ becomes $\sim 0.86$. With filters with much narrower bandwidths, $\left|\gamma_{0}\right|^{2}$ approaches unity. Substituting $\left|\gamma_{0}\right|^{2}=0.86$ in Eq. (43) with $|\alpha|^{2}=1$ and $\eta=0.5$ gives a fidelity value of $F \simeq 0.82$. When $|\alpha|^{2}=0.5$ is used, a value of $F \simeq 0.89$ is obtained.

Although in the above discussion, we have analyzed only the temporal mode matching, the expressions for the spatial mode matching can be derived using the same procedure. It must also be noted that using very narrow frequency and spatial filters will result in attenuation of the fields incident on the detectors causing a decrease in the rate of having a correct detection. In a realistic experiment scheme, like the one we have proposed in [9], very good spatial mode matching can be achieved by using single-spatial-mode fibers after BS2 when the output modes are input to the photon-counting detectors 26 28]. A spatiotemporal mode matching value of $\sim 0.66$ has been reported in a quantum tomography of single-photon-state experiment [25]. Rarity et al. 28] have reported an experimentally obtained visibility of $\sim 0.63$ in an experimental scheme similar to our proposal [9]. In another experiment performed to test Belltype inequality for EPR state in a homodyne measurement, Kuzmich et al. 26 have reported visibility values greater than 0.8 by using narrow-band filters with bandwidths $3.5 \mathrm{~nm}$ and $6 \mathrm{~nm}$. Within the range of reported experimental values for $\left|\gamma_{0}\right|^{2}$, we can predict a fidelity of $F \geq 0.7$ for state truncation and preparation using the QSD scheme when $|\alpha|^{2} \leq 1.0$, i.e with $\left|\gamma_{0}\right|^{2}=0.66$, $\eta=0.5$ and $|\alpha|^{2}=0.5$, a fidelity value of $\sim 0.8$ is calculated. Higher values of $|\alpha|^{2}$ will reduce the attainable fidelity. 


\section{CONCLUSION}

A major obstacle for the practical realization of state truncation and preparation using projection synthesis and quantum-scissors device is the mode mismatch of the input lights to the device. In order to study this problem and its effect on the quality of the process we have developed the pulse-mode projection synthesis, characterize the mode of the interfering lights, and derived the analytical expressions for the output density matrix and fidelity. The study includes not only the mode mismatch between the interfering lights but that between them and the photodetectors, as well. POVMs for the analysis are derived and discussed. It has been understood that mode mismatch destroys the off-diagonal elements of the output density matrix strongly and in the limiting case of complete mismatch, off-diagonal elements become zero resulting in a classical mixture at the output. When the intensity of the input coherent light is much lower than one, mode mismatch and detector efficiency do not have significant effect on the output of the process. When the intensity becomes higher, fidelity of the truncation process degrades rapidly with increasing mismatch. The same behavior is shown to be valid for the preparation of arbitrary superpositions of vacuum and one-photon states. It has been depicted that the intensity of the input coherent light can always be optimized to maximize the fidelity of the preparation of a desired superposition state. When desired state has vacuum component dominant, then effect of mismatch is not significant, however when one-photon state becomes dominant fidelity is strongly affected by mismatch. In low modemismatch cases, increasing detection efficiency increases the fidelity of truncation, however, when the mode mismatch becomes larger, the effect of detector efficiency on the fidelity of the process decreases.

\section{ACKNOWLEDGMENTS}

We thank Takashi Yamamoto and Yu-xi Liu for stimulating discussions.

\section{REFERENCES}

[1] Special issue on Quantum State Preparation and Measurement, J. Mod. Opt. 44 (11/12) (1997).
[2] D.T. Pegg, L.S. Phillips and S.M. Barnett, Phys. Rev. Lett 81, 1604, (1998).

[3] M. Dakna, J. Clausen, L. Knöll, and D. G. Welsch, Phys. Rev. A 59, 1658 (1999) and references therein.

[4] G. M. D'Ariano, L. Maccone, M. G. A. Paris, and M. F. Sacchi, Phys. Rev. A 61, 053817 (2000).

[5] S.M. Barnett and D. T. Pegg, Phys. Rev. Lett. 76, 4148, (1996).

[6] B. Baseia, M.H.Y. Moussa, and V.S. Bagnato, Phys. Lett. A 231, 331, (1997).

[7] L.S. Phillips, S.M. Barnett, and D.T. Pegg, Phys. Rev. A 58, 3259 (1998).

[8] S.M. Barnett, and D.T. Pegg, Phys. Rev. A 60, 4965 (1999).

[9] Ş.K. Özdemir, A. Miranowicz, M. Koashi, and N. Imoto, Phys. Rev. A 64, 063818 (2001).

[10] M. Koniorczyk, Z. Kurucz, A. Gabris, and J. Janszky, Phys. Rev. A 62, 013802 (2000).

[11] C. J. Villas-Boas, Y. Guimarães, M. H. Y. Moussa, and B. Baseia, Phys. Rev. A 63, 055801 (2001). (2001)

[12] M. G. A. Paris, Phys. Rev. A 62, 033813 (2000).

[13] Ş.K. Özdemir, A. Miranowicz, M. Koashi, and N. Imoto, J. Mod. Opt. 49, 977 (2002).

[14] Y. Guimarães, B. Baseia, C. J. Villas-Boas, and M. H. Y. Moussa, Phys. Lett. A 268, 260, (2000).

[15] C. J. Villas-Bôas, N. G. de Almeida, and M. H. Y. Moussa, Phys. Rev. A 60, 2759, (1999).

[16] K. J. Blow, R. Loudon, S. J. D. Phoenix, and T. J. Shepherd, Phys. Rev. A. 42, 4102 (1990).

[17] N. Hussain, N. Imoto, and R. Loudon, Phys. Rev. A 45, 1987 (1992).

[18] N. Imoto, in Quantum Physics, Chaos Theory, and Cosmology, ed. M. Namiki et al., (AIP Press, New York, 1996) p.173.

[19] D. J. Santos, R. Loudon, and F .J . Fraile-Peláez, Am. J. Phys. 65, 126 (1997),

[20] R. A. Campos, B. E. A. Saleh, and M. C. Teich, Phys. Rev. A 40, 1371 (1989).

[21] S. M. Barnett, L. S. Phillips, and D. T. Pegg, Opt. Commun. 158, 45 (1998).

[22] Z. Y. Ou, L. J. Wang, and L. Mandel, Phys. Rev. A 40, 1428 (1989).

[23] Z. Y. Ou, Qu. Semiclass. Opt. 9, 599 (1997).

[24] W. P. Grice, and I. A. Walmsley, Phys. Rev. A 56, 1627 (1997).

[25] T. Aichele, A. I. Lvovsky, and S. Schiller, Eur. Phys. J. D 18, 237 (2002).

[26] A. Kuzmich, I. A. Walmsley, and L. Mandel, Phys. Rev. A 64, 063804 (2001).

[27] J. G. Rarity, P. R. Tapster, and R. Loudon, e-print quan$\mathrm{ph} / 9702032$.

[28] J. G. Rarity, P. R. Tapster, and R. Loudon, in Quantum Interferometry, ed. F. de Martini, et al. (VCH Press, New York, 1996) p. 211. 